\title{
Some Asymptotic Estimates of Transition Probability Densities for Generalized Diffusion Processes with Self-similar Speed Measures
}

By

\author{
Takahiko FuנITA*
}

\section{§ 1. Introduction}

To a non-negative Borel measure $d m(x)$ on an interval with suitable boundary conditions on the end points, we can associate a generalized differential operator $A=\frac{d}{d m(x)} \frac{d}{d x}$ and a strong Markov process $X$ on the support of $d m$ generated by the operator $A$. The measure $d m$ is often called $a$ string and the process $X$ a generalized diffusion, also a quasi-diffusion or a gap diffusion, with the speed measure $d m(x)$, cf. [9] for details.

Let $0 \geq \lambda_{1}>\lambda_{2} \geq \cdots$ be the eigenvalues of $A=\frac{d}{d m(x)} \frac{d}{d x}$ and let $p(t, x, y)$ be the transition probability density of $X$ with respect to $d m(x)$. It was shown by M.G. Krein [10] and H.P. Mckean-D.B. Ray [12] that

$$
\lim _{n \rightarrow \infty} \frac{-\lambda_{n}}{n^{2}}=\left(\frac{1}{\pi} \int_{0}^{1} \sqrt{\frac{d m}{d x}(x)} d x\right)^{-2} .
$$

Also it was shown by S. Watanabe ([9], Appendix 2) that

$$
\lim _{t \rightarrow 0}(-2 t) \log p(t, x, y)=\left(\int_{x}^{y} \sqrt{\frac{1}{2} \frac{d m}{d x}(x)} d x\right)^{2} .
$$

Here in (1.1) and (1.2), $\frac{d m}{d x}(x)$ denotes the Radon-Nikodym density of the absolutely continuous part of the measure $d m(x)$. In the case when $d m(x)$ is singular, therefore, (1.1) implies only that $-\lambda_{n}$ grows faster than $n^{2}$ and (1.2)

Communicated by S. Matsuura, November 9, 1989.

* Department of Mathematics, Kyoto University, Kyoto 606, Japan. 
implies only that $-\log p(t, x, y)$ grows slower than $t^{-1}$ as $t \rightarrow 0$. Thus one is naturally lead, in this case, to a problem of finding more exact growth orders of $-\lambda_{n}$ and $-\log p(t, x, y)$.

In the previous paper [3], we obtained an estimate for the growth order of eigenvalues for generalized differential operators associated with certain selfsimilar measures $d m(x)$. Here we follow Hutchinson [5] for relevant notions on the self-similarity: He defined the self-similarity of sets and measures by a family of contraction affine maps and succeeded in giving a solid foundation of the fractal theory of B.B. Mandelbrot [11]. Such self-similar measures include, even in the one-dimensional case, many interesting examples of singular measure like the Cantor measure and the de Rham measure.

The main purpose of this paper is to obtain more exact estimates for $-\log p(t, x, y)$ in the case of self-similar measures $d m(x)$. As an application of our result, we can give an example of some generalized diffusion processes which do not have Barlow-Perkins type estimates (see [1]). Furthermore, in the last part of $\S 3$, we can have some supplementary remarks on our previous paper [3] which give some relations between the spectral dimension, the entropy and the Kolmogoroff dimension.

The author expresses his heartfelt gratitude to Prof. S. Watanabe and Prof. S. Kotani for their valuable suggestions.

\section{§. Preliminaries}

Before proceeding, we have to recall some basic facts on Krein's spectral theory of strings (cf. [9] for details).

Take a non-negative Borel measure $d m(x)$ on $[0, a](0<a \leq+\infty)$ such that its restriction to $[0, b), b<a$, is a Radon measure.

Let $\phi(x, \lambda)$ and $\psi(x, \lambda)$ be continuous solutions of

$$
\phi(x, \lambda)=1+\lambda \int_{0}^{x}(x-y) \phi(x, \lambda) d m(y)
$$

and

$$
\psi(x, \lambda)=x+\lambda \int_{0}^{x}(x-y) \psi(x, \lambda) d m(y)
$$

for $0 \leq x<a$.

Set

$$
h(\lambda)=\int_{0}^{a} \frac{d x}{\phi(x, \lambda)^{2}}=\lim _{x \uparrow^{a}} \frac{\psi(x, \lambda)}{\phi(x, \lambda)}
$$

$h(\lambda)$ is called the characteristic funcition of $d m$ and the one-to-one correspondence 
$h \leftrightarrow m$ is called Krein's correspondence. Then, following results are well known (see [9]):

(Comparison theorem) Let $m_{1}, m_{2}$ be two measures and $h_{1}, h_{2}$ be the corresponding characteristic functions of $m_{1}, m_{2}$ respectively. If $m_{1}(x) \leq m_{2}(x)$ for all $x>0$, then $h_{1}(\lambda) \geq h_{2}(\lambda)$ holds.

(Kac's inequality) If $h$ is the characteristic function of $m$,

$$
\frac{1}{\lambda m([0, x))+\frac{1}{x}} \leq h(\lambda) \leq x+\frac{1}{\lambda m([0, x))} \quad \text { for } \quad \lambda>0, x>0 \text {. }
$$

(A corollary to Kac's inequality) Let $u(x)$ be the inverse function of $x m([0, x)$ ). Then

$$
\frac{1}{2} u\left(\frac{1}{\lambda}\right) \leq h(\lambda) \leq 2 u\left(\frac{1}{\lambda}\right) \quad \text { for } \quad \lambda>0 \text {. }
$$

\section{§ 3. Self-similar Sets, Self-similar Measures and Eigenvalue Problems}

Self-similar sets and self-similar measures are introduced by Hutchinson [5]. In this section, we first give a brief review of Hutchinson's setting that is essentially required to our theory. For simplicity, we state his theory in the one dimensional case. Let $S_{i}(i=1, \cdots N)$ be contraction affine maps from $[0,1]$ to $[0,1]$ i.e. $S_{i}(x)=r_{i} x+b_{i}$ where $-1<r_{i}<1,0 \leq b_{i} \leq 1$, and $0 \leq r_{i}+b_{i} \leq 1$.

Definition. A compact set $K(\subset[0,1])$ is called the self-similar set with respect to $S=\left\{S_{1}, \cdots, S_{N}\right\}$ (or simply the $S$-self similar set) if $K=\bigcup_{i=1}^{N} S_{i} K$.

Definition. Suppose $\rho=\left(\rho_{1}, \cdots, \rho_{N}\right)$ where $\rho_{1}, \cdots, \phi_{N} \in(0,1)$ and $\sum_{i=1}^{N} \rho_{i}=1$. A measure $m$ is called the self-similar measure with respect to $S$ and $\rho$ (or simply the $(S, \rho)$ self-similar measure) if $m(A)=\sum_{i=1}^{N} \rho_{i} m\left(S_{i}^{-1}(A)\right)$ for any Borel set $A$ $(\subset[0,1])$.

Then, by Hutchinson [5], it is known that there exists uniquely the $S$-selfsimilar set which we denote by $K(S)$, and the $(S, \rho)$ self-similar measure which we denote by $\mu(S, \rho)$ and that the topological support of $\mu(S, \rho)$ coincides with $K(S)$.

Definition. Given $S$ and $\rho$ as above, the unique number $s(0<s \leq 1)$ such 
that $\sum_{i=1}^{N}\left(\rho_{i}\left|r_{i}\right|\right)^{s /(1+s)}=1$ is called the similarity dimension of $\mu(S, \rho)$.

This $s$ is introduced in [3] and describes the asymptotic order of eigenvalues for generalized second order differential operator associated with $m=\mu(S, \rho)$. Namely we obtained the following results.

For $0 \leq \alpha, \beta \leq \frac{\pi}{2}, 0 \leq a<b \leq 1$, consider the following eigenvalue problems of $L=\frac{d}{d m} \frac{d}{d x}$ on $[0,1]$ :

$$
\begin{aligned}
& L f=\lambda f \text { in }(0,1) \\
& f(0) \cos \alpha-\frac{d}{d x} f(0) \sin \alpha=0 \\
& f(1) \cos \beta+\frac{d}{d x} f(1) \sin \beta=0 .
\end{aligned}
$$

Theorem 3.1. ([3]). Let $S=\left\{S_{1}, \cdots, S_{N}\right\}$ and $\rho=\left(\rho_{1}, \cdots, \rho_{N}\right)$ satisfying that $S_{i}[0,1] \cap S_{j}[0,1]=\{$ one point $\}$ or $\phi$ for $i \neq j$.

Consider the eigenvalue problem (3.1) and let $\lambda_{n}$ be eigenvalues such that $0 \geq \lambda_{1}>\lambda_{2} \geq \lambda_{3}, \cdots$. Then there exists positive constans $C_{1}, C_{2}$ and $n_{0}$ such that

$$
C_{1} n^{(1+s) / s}<-\lambda_{n}<C_{2} n^{(1+s) / s} \quad \text { for any } n \geq n_{0},
$$

where $s$ is the similarity dimension of $\mu(S, \rho)$.

If $\lim _{n \rightarrow \infty} \frac{\log -\lambda_{n}}{\log n}(=t)$ exists, this theorem suggests that we may call $d=\frac{1}{t-1}$ $\in[0,1]$ the spectral dimension of the measure $m$. Theorem 3.1. asserts that $d=s$.

Example (Cantor function). If we take $N=2, S_{1}(x)=\frac{x}{3}, S_{2}(x)=\frac{x+2}{3}, S=$ $\left\{S_{1}, S_{2}\right\}, \rho=\left(\frac{1}{2}, \frac{1}{2}\right)$,

then $\quad K(S)=$ the triadic Cantor set

and $\mu(S, \rho)=$ the Cantor measure (a probability measure corresponding to Cantor function). In this case, $s=\frac{\log 2}{\log 3}(=$ the Hausdorff dimension of $K(S))$. Theorem 3.1.im-
plies that

$$
C_{1} n^{\log 6 / \log 2}<-\lambda_{n}<C_{2} n^{\log 6 / \log 2} \text { as } n \rightarrow \infty
$$

where $C_{1}, C_{2}$ are positive constants (see [3], [4], [12]). Similarly, taking $f_{1}(x)=$ $r x+b_{1}, \cdots, f_{N}(x)=r x+b_{N}$ such that $(*)$ holds i.e. $0<b_{1}<r+b_{1}<b_{2}<r+b_{2}<\cdots$ 
$<r+b_{N-1}<b_{N}<r+b_{N}<1$ and $\rho=\left(\frac{1}{N}, \cdots, \frac{1}{N}\right)$,

then $\quad K(S)=$ a generalized Cantor set

and $\mu(S, \rho)=$ a generalized Cantor measure

In this case $s=\frac{\log \frac{1}{N}}{\log r}$, so we see that

$$
C_{1} n^{\log (r / N) / \log (1 / N)}<-\lambda_{n}<C_{2} n^{\log (r / N) / \log (1 / N)} \text { as } n \rightarrow \infty
$$

where $C_{1}$ and $C_{2}$ are positive constants.

Example. (de Rham function [13] or Bernoulli trial for unfair coin).

If we take $N=2, S_{1}(x)=\frac{x}{2}, S_{2}(x)=\frac{x+1}{2}, S=\left\{S_{1}, S_{2}\right\}, \rho=(p, q)(p+q=1, p>0$, $q>0, p \neq q)$

then $\quad K(S)=[0,1]$

and $\mu(S, \rho)=$ the de Rham measure (a probability measure corresponding to the de Rham function $F$ i.e.

$$
F(x)=P\left(\omega \mid \sum_{n=1}^{\infty} \frac{X_{n}(\omega)}{2^{n}} \leq x\right) \text { where } X_{n}:\{0,1\} \text { valued i.i.d }
$$

random variables such that $P\left(X_{n}=1\right)=p, P\left(X_{n}=0\right)=q$. In this case, if $\alpha$ is the unique number such that $\left(\frac{p}{2}\right)^{\alpha}+\left(\frac{q}{2}\right)^{\alpha}=1$ then, $s=\frac{\alpha}{1-\alpha}$ and $C_{1} n^{1 / \alpha}<-\lambda_{n}<$ $C_{2} n^{1 / \alpha}$ as $n \rightarrow \infty$ for some positive constants $C_{1}$ and $C_{2}$.

In the rest of this section, we consider the following problem as a supplement to our previous paper [3]: We want to estimate the spectral dimension of $d m$ by other fractional dimensions from upper and lower sides. First, we consider a lower estimate. In the de Rham measure case, we obtained that ([3])

$$
s \geq-p \log _{2} p-q \log _{2} q=\text { the entropy of } B(p, q)
$$

where $B(p, q)$ is the $(p, q)$-Bernoulli shift. We can prove that (3.2) holds in a more general situation.

Proposition 3.2. Let $X_{i}(i=1,2, \cdots \cdots)$ be a discrete time Markov chain with a finite number of states $0,1, \cdots, M-1$ and consider the random variable $X=$ $\sum_{i=1}^{\infty} X_{i} M^{-i}$ with distribution $F: F([0, x])=P(X \leq x)$. Then, it holds that the entropy of $F \leq$ the spectral dimension of $F$. 
Proof. As a version of Shannon-McMillan theorem, J.R. Kinney [8] showed the following: There exists a set $E \subset[0,1]$ such that (1) $F(E)=1$, (2) the Haussdorff dimension of $E=\alpha$, and (3) if $x \in E$ and $\varepsilon>0$, then

$$
\lim _{h \downarrow 0} \frac{F(x-h, x+h)}{h^{\alpha-\varepsilon}}=0, \lim _{h \downarrow 0} \frac{F(x-h, x+h)}{h^{\alpha+\varepsilon}}=+\infty
$$

where $\alpha$ is the entropy of $F\left(=-\sum p_{i} p_{i j} \log p_{i j}\right), p_{i j}$ the transition probability and $p_{i}$ the stationary probability. When $x \in E$, consider

$$
m_{+}^{x}(\varepsilon)=F([x, x+\varepsilon)), \quad m_{-}^{x}(\varepsilon)=F((x-\varepsilon, x))
$$

and take corresponding characteristic functions $h_{+}^{x}(\lambda), h_{-}^{x}(\lambda)$ respectively in Krein's correspondence. Then, applying (2.2),

$$
\begin{aligned}
& \left\{\begin{array}{l}
\frac{1}{2} U_{+}^{x}\left(\frac{1}{\lambda}\right) \leq h_{+}^{x}(\lambda) \leq 2 U_{+}^{x}\left(\frac{1}{\lambda}\right) \\
\frac{1}{2} U_{-}^{x}\left(\frac{1}{\lambda}\right) \leq h_{-}^{x}(\lambda) \leq 2 U_{-}^{x}\left(\frac{1}{\lambda}\right)
\end{array}\right. \\
& \text { (3.5) } U_{+}^{x}\left(\frac{1}{\lambda}\right) m_{+}^{x}\left(U_{+}^{x}\left(\frac{1}{\lambda}\right)\right)=1 \text { and } U_{-}^{x}\left(\frac{1}{\lambda}\right) m_{-}^{x}\left(U_{-}^{x}\left(\frac{1}{\lambda}\right)\right)=1 .
\end{aligned}
$$

By (3.3) and (3.5), we have for every positive $\delta$,

$$
\begin{aligned}
0 & =\lim _{\lambda \rightarrow \infty} \frac{m_{+}^{x}\left(U_{+}^{x}\left(\frac{1}{\lambda}\right)\right)}{U_{+}^{x}\left(\frac{1}{\lambda}\right)^{\alpha-\delta}} \\
& =\lim _{\lambda \rightarrow \infty} \frac{1}{\lambda U_{+}^{x}\left(\frac{1}{\lambda}\right)^{1+\infty-\delta}} .
\end{aligned}
$$

Then,

$$
\lim _{\lambda \rightarrow \infty} \frac{1}{\lambda^{1 /(1+\infty-\delta)} U_{+}^{x}\left(\frac{1}{\lambda}\right)}=0
$$

In the same way, we have

$$
\lim _{\lambda \rightarrow \infty} \frac{1}{\lambda^{1 /(1+\infty-\delta)} U_{-}^{x}\left(\frac{1}{\lambda}\right)}=0
$$

Let $g_{\lambda}(x, x)$ be the Green kernel of $\frac{d}{d F} \frac{d}{d x}$ with suitable boundary conditions, 
i.e. $g_{\lambda}(x, y)=\int_{0}^{\infty} p(t, x, y) d t$ where $p(t, x, y)$ is the transition probability density with respect to $F$ of the corresponding diffusion. Then, it is well known that

$$
g_{\lambda}(x, x)=\frac{1}{\frac{1}{h_{+}^{x}(\lambda)}+\frac{1}{h_{-}^{x}(\lambda)}} .
$$

By (3.4) and (3.7), we see that

$$
\int_{E} g_{\lambda}(x, x) d F(x) \geq \frac{1}{2} \int_{E} \frac{1}{\frac{1}{U_{+}^{x}\left(\frac{1}{\lambda}\right)}+\frac{1}{U^{x}-\left(\frac{1}{\lambda}\right)}} d F(x) .
$$

Seeing (3.6), (3.7) and (3.9), we have for every positive $\delta$,

$$
\begin{aligned}
& \varliminf_{\lambda \rightarrow \infty} \lambda^{1 /(1+\infty-\delta)} \int_{E} g_{\lambda}(x, x) d F(x) \\
& \geq \frac{1}{2} \int_{E} \frac{\lim _{\lambda \rightarrow \infty}}{\frac{1}{\lambda^{1 /(1+\infty-\delta)} U_{+}^{x}\left(\frac{1}{\lambda}\right)}+\frac{1}{\lambda^{1 /(1+\alpha-\delta)} U^{x}\left(\frac{1}{\lambda}\right)}} d F(x) \\
& \quad=+\infty
\end{aligned}
$$

On the other hand, the definition of the spectral dimension $d$ and Tauberian theorem show that

$$
-\frac{1}{1+d}=\lim _{\lambda \rightarrow \infty} \frac{1}{\log \lambda} \log \int_{E} g_{\lambda}(x, x) d F(x) .
$$

Then (3.10) and (3.11) imply that for every positive $\delta$,

$$
\frac{1}{1+\alpha-\delta}-\frac{1}{1+d} \geq 0
$$

So we have $d \geq \alpha-\delta$ for every positive $\delta$.

Thus the proof of this proposition is complete.

Next, we consider an upper estimate. Let $K$ be a compact metric space. We denote by $\bar{h}(K)$ the upper Kolmogoroff dimension of $K$ i.e.

$$
\bar{h}(K)=\varlimsup_{\varepsilon_{\downarrow} 0} \frac{\log N_{\mathrm{\varepsilon}}}{\log \frac{1}{\varepsilon}}
$$


where $N_{\mathrm{z}}=$ the infimum of the number of $\varepsilon$-cover of $K$.

Proposition 3.3. We take $d m=d \mu(S, \rho)$ as in Theorem 3.1. Then it holds that the spectral dimension of $d m \leq \bar{h}(K(S))$.

Proof. By (3.8) and Kac's inequality, we have for every positive $\varepsilon$,

$$
\begin{aligned}
g_{\lambda}(x, x)=\frac{1}{\frac{1}{h_{+}^{x}(\lambda)}+\frac{1}{h_{-}^{x}(\lambda)}} & \leq \frac{1}{\varepsilon+\frac{1}{\lambda m[x, x+\varepsilon)}+\frac{1}{\varepsilon+\frac{1}{\lambda m[x-\varepsilon, x)}}} \\
& \leq 2 \varepsilon+\frac{1}{\lambda m(x-\varepsilon, x+\varepsilon)} .
\end{aligned}
$$

Then we have that

$$
\int_{0}^{1} g_{\lambda}(x, x) d m(x) \leq 2 \varepsilon+\frac{1}{\lambda} \int_{0}^{1} \frac{d m(x)}{m(x-\varepsilon, x+\varepsilon)} .
$$

Define

$$
\alpha(m)=\varlimsup_{\varepsilon_{\downarrow}} \frac{1}{\log \frac{1}{\varepsilon}} \log \int_{0}^{1} \frac{d m(x)}{m(x-\varepsilon, x+\varepsilon)} .
$$

For every positive $\delta$, there exists $\varepsilon_{0}$ such that

$$
\int_{0}^{1} \frac{d m(x)}{m(x-\varepsilon, x+\varepsilon)} \leq\left(\frac{1}{\varepsilon}\right)^{\alpha(m)+\delta} \quad \text { for all } 0<\varepsilon<\varepsilon_{0} .
$$

Then, $\quad \int_{0}^{1} g_{\lambda}(x, x) d m(x) \leq 2 \varepsilon+\frac{1}{\lambda}\left(\frac{1}{\varepsilon}\right)^{\alpha(m)+\delta}$.

Taking $\quad \frac{1}{\lambda}=2 \varepsilon^{1+\infty(m)+\delta}$,

$$
\int_{0}^{1} g_{\lambda}(x, x) d m(x) \leq 4\left(\frac{1}{2 \lambda}\right)^{1 /(1+\alpha(m)+\delta)}
$$

Then, $\quad \varlimsup_{\lambda \rightarrow \infty} \frac{1}{\log \lambda} \log \int_{0}^{1} g_{\lambda}(x, x) d m(x) \leq-\frac{1}{1+\alpha(m)+\delta}$.

Since $\delta$ is arbitrary positive, it holds that

$$
-\frac{1}{1+d} \leq-\frac{1}{1+\alpha(m)} \text { i.e. } d \leq \alpha(m)
$$

where $d$ is the spectral dimension of $d m$. On the otherh and, we take $U_{i}(i=1$, $\cdots, N)$ as a $\frac{\varepsilon}{4}$-cover of $K(S)$. Then we have that 


$$
\begin{aligned}
\int_{K} \frac{d m(x)}{m(x-\varepsilon, x+\varepsilon)} & \leq \sum_{i=1}^{N} \int_{U_{i}} \frac{d m(x)}{m(x-\varepsilon, x+\varepsilon)} \\
& \leq \sum_{1=i}^{N} \int_{U_{i}} \frac{d m(x)}{m\left(U_{i}\right)}=N
\end{aligned}
$$

because $x \in U_{i}$ implies $U_{i} \subset(x-\varepsilon, x+\varepsilon)$.

Then $\alpha(m)=\varlimsup_{\varepsilon_{\downarrow}} \frac{1}{\log \frac{1}{\varepsilon}} \log \int_{K} \frac{d m(x)}{m(x-\varepsilon, x+\varepsilon)}$

$$
\begin{aligned}
& \leq \varlimsup_{\varepsilon \downarrow 0} \frac{\text { the infimum of the number of } \frac{\varepsilon}{4} \text {-cover of } K}{\log \frac{1}{\varepsilon}} \\
& =\bar{h}(K) .
\end{aligned}
$$

Therefore, combining this with (3.12), we have the assertion.

Q.E.D.

Remark. In the Cantor measure case, $\alpha=d=s=\bar{h}$.

But, in the de Rham measure case, $\alpha<d=s<\bar{h}$.

\section{§ 4. Asymptotic Estimates of Transition Probability Densities}

In this section, we discuss some asymptotic estimates of transition densities. First, using Kac's inequality, we prepare some basic lemmas.

Take positive numbers $a, b$. Let $d m(x)$ be a bounded measure on $(-a, b)$ and let $L$ be $\frac{d}{d m(x)} \frac{d}{d x}$. We denote by $\bar{E}_{x}^{\alpha}(\quad)$ the expectation with respect to the L-generalized diffusion processes on $[0, b)$ starting from $x(0 \leq x<b)$ with boundary conditions $f(0) \cos \alpha-f^{\prime}(0) \sin \alpha=0$ for some $\alpha \in\left(0, \frac{\pi}{2}\right]$ and $f(b)=0$. We also denote by $E_{x}^{a^{\prime}}($ ) the expectation with respect to the L-generalized diffusion processes on $(-a, b)$ starting from $x(-a \leq x<b)$ with boundary conditions $f(-a) \cos \alpha^{\prime}-f^{\prime}(-a) \sin \alpha^{\prime}=0$ for some $\alpha^{\prime} \in\left(0, \frac{\pi}{2}\right]$ and $f(b)=0$.

Lemma 4.1. Let $\tau_{c}=\inf \left\{t>0 \mid X_{t}=c\right\}$ for $0<c<b$. Then

$$
\begin{aligned}
& \frac{1}{1+\cot \alpha\left(b+\frac{1}{\lambda m([0, b)}\right)} \leq \frac{\bar{E}_{0}^{\alpha} e^{-\tau_{c}}}{E_{0}^{\alpha^{\prime}} e^{-\tau_{c}}} \\
& \leq 1+\frac{b}{a}+\frac{m((-a, 0))}{m([0, b))}+\frac{1}{\lambda a m([0, b))}+\lambda m((-a, 0))
\end{aligned}
$$

for every positive $\lambda$. 
Proof. First, we prove the first inequality of (4.1). Let

$$
\begin{array}{ll}
m_{1}(x)=m_{1}([0, x)) & \text { for } \quad x<b \\
m_{2}(x)=m_{2}((-x, 0)) & \text { for } \quad x<a
\end{array}
$$

and $h_{1}, h_{2}$ be the corresponding characterestic functions respectively. Let

and

$$
\begin{aligned}
& \varphi_{i}(x)=1+\int_{0}^{x}(x-y) \varphi_{i}(y) d m_{i}(y) \\
& \psi_{i}(x)=x+\int_{0}^{x}(x-y) \psi_{i}(y) d m_{i}(y) \\
& \varphi(x, \lambda)= \begin{cases}\varphi_{1}(x, \lambda) & \text { for } 0 \leq x<b \\
\varphi_{2}(-x, \lambda) & \text { for }-a<x<0\end{cases} \\
& \psi(x, \lambda)= \begin{cases}\psi_{1}(x, \lambda) & \text { for } 0 \leq x<b \\
-\psi_{2}(-x, \lambda) & \text { for }-a<x<0 .\end{cases}
\end{aligned}
$$

Then it is well known (see [6]) that, there exists some constants $C_{1}, C_{2}$ such that

$$
E_{x}^{\alpha^{\prime}} e^{-\lambda \tau_{c}}=\frac{C_{1} \varphi(x, \lambda)+C_{2} \psi(x, \lambda)}{C_{1} \varphi(c, \lambda)+C_{2} \psi(c, \lambda)} \quad \text { for } \quad x<c
$$

Considering the boundary condition at $-a$, we have

$$
\begin{aligned}
0 & =\left\{C_{1} \varphi(-a, \lambda)+C_{2} \psi(-a, \lambda)\right\} \cos \alpha^{\prime}-\left\{C_{1} \varphi^{\prime}(-a, \lambda)+C_{2} \psi^{\prime}(-a, \lambda)\right\} \sin \alpha^{\prime} \\
& =\left\{C_{2} \varphi_{2}(a, \lambda)-C_{2} \psi(a, \lambda)\right\} \cos \alpha^{\prime}-\left\{-C_{1} \varphi_{2}^{\prime}(a, \lambda)+C_{2} \psi_{2}^{\prime}(a, \lambda)\right\} \sin \alpha^{\prime} .
\end{aligned}
$$

Then

$$
\frac{C_{2}}{C_{1}}=\frac{\varphi_{2}(a, \lambda) \cos \alpha^{\prime}+\varphi_{2}^{\prime}(a, \lambda) \sin \alpha^{\prime}}{\psi_{2}(a, \lambda) \cos \alpha^{\prime}+\psi_{2}^{\prime}(a, \lambda) \sin \alpha^{\prime}} .
$$

So we have that

$$
\begin{gathered}
E_{0}^{\alpha^{\prime}} e^{-\lambda \tau_{c}}=\frac{1}{\varphi_{1}(c, \lambda)+\frac{\varphi_{2}(a, \lambda) \cos \alpha^{\prime}+\varphi_{2}^{\prime}(a, \lambda) \sin \alpha^{\prime}}{\psi_{2}(a, \lambda) \cos \alpha^{\prime}+\psi_{2}^{\prime}(a, \lambda) \sin \alpha^{\prime}} \psi_{1}(c, \lambda)} \\
\bar{E}_{0}^{\alpha} e^{-\lambda \tau_{c}}=\frac{1}{\varphi_{1}(c, \lambda)+\cot \alpha \psi_{1}(c, \lambda)} .
\end{gathered}
$$

By (4.2), (4.3) and Kac's inequality, we obtain that

$$
\begin{aligned}
\frac{\bar{E}_{0}^{\alpha} e^{-\lambda \tau_{c}}}{E_{0}^{\alpha^{\prime}} e^{-\lambda \tau_{c}}} & =\frac{\varphi_{1}(c, \lambda)+\frac{\varphi_{2}(a, \lambda) \cos \alpha^{\prime}+\varphi_{2}^{\prime}(a, \lambda) \sin \alpha^{\prime}}{\psi_{2}(a, \lambda) \cos \alpha^{\prime}+\psi_{2}^{\prime}(a, \lambda) \sin \alpha^{\prime}} \psi_{1}(c, \lambda)}{\varphi_{1}(c, \lambda)+\cot \alpha \psi_{1}(c, \lambda)} \\
& \geq \frac{1}{1+\cot \alpha \frac{\psi_{1}(c, \lambda)}{\varphi_{1}(c, \lambda)}} \cdot
\end{aligned}
$$


The proof of the first inequality of (4.1) is complete. In the same way, we have

$$
E_{0}^{0} e^{-\lambda \tau_{c}}=\frac{1}{\varphi_{1}(c, \lambda)+\frac{1}{h_{2}(\lambda)} \psi_{1}(c, \lambda)}
$$

and

$$
\bar{E}_{0}^{\pi / 2} e^{-\lambda \tau_{c}}=\frac{1}{\varphi_{1}(c, \lambda)}
$$

Then

$$
\begin{aligned}
& \frac{\bar{E}_{0}^{\alpha} e^{-\lambda \tau_{b}}}{E_{0}^{\alpha} e^{-\lambda \tau_{c}}} \leq \frac{\bar{E}_{0}^{\pi / 2} e^{-\lambda \tau_{c}}}{E_{0}^{0} e^{-\lambda \tau_{c}}} \\
&=1+\frac{1}{h_{2}(\lambda)} \frac{\psi_{1}(c, \lambda)}{\varphi_{1}(c, \lambda)} \\
& \leq 1+\frac{1}{h_{2}(\lambda)} h_{1}(\lambda) .
\end{aligned}
$$

Applying Kac's inequality (2.1) again we obtain the second inequality of (4.1).

Q.E.D.

We also need the following:

Lemma 4.2. If $m([0, b))=0$, then

$$
1 \geq E_{0}^{a} e^{-\lambda \tau_{c}} \geq \frac{1}{1+\lambda b m((-a, 0))+\frac{b}{a}} \quad \text { for } 0 \leq c<b .
$$

Proof. The first inequality is trivial. As for the second,

$$
\begin{aligned}
E_{0}^{\alpha} e^{-\lambda \tau_{c}} & \geq E_{0}^{0} e^{-\lambda \tau_{c}} \\
& =\frac{1}{\varphi_{1}(c, \lambda)+\frac{1}{h_{2}(\lambda)} \psi_{1}(c, \lambda)} \\
& =\frac{1}{1+\frac{c}{h_{2}(\lambda)}} \geq \frac{1}{1+\frac{b}{h_{2}(\lambda)}} .
\end{aligned}
$$

By Kac's inequality (2.1) we obtain our lemma.

Q.E.D.

Take $m=\mu(S, \rho)$ where $S=\left\{S_{1}, \cdots, S_{N}\right\}, \rho=\left(\rho_{1}, \cdots, \rho_{N}\right)$ such that $S_{i}(x)=$ $r_{i} x+c_{i},-1<r_{i}<1$ for $1 \leq i \leq N$. Putting $S_{i}([0,1])=\left[a_{i}, b_{i}\right]$, we assume $0 \leq a_{1} \leq$ $b_{1} \leq \cdots \leq a_{N} \leq b_{N} \leq 1$. Let us consider $L=\frac{d}{d m(x)} \frac{d}{d x}$-generalized diffusion process $X_{t}$ on $[0,1]$ with boundary conditions

$$
f(0) \cos \alpha-f^{\prime}(0) \sin \alpha=0 \text { and } f(1) \cos \beta+f^{\prime}(1) \sin \beta=0
$$




$$
\text { for } 0<\alpha, \beta<\frac{\pi}{2} \text {. }
$$

Let $p(t, x, y)$ be the transition probability density of $X_{t}$ with respect to $d m$.

Theorem 4.3. Take $x, y$ such that $0<x<y<1$ and $m([x, y])>0$. Then, there exists positive constants $C_{4.1}, C_{4.2}$ which depend on $x, y$ such that

$$
C_{4.1} t^{-s} \leq-\log p(t, x, y) \leq C_{4.2} t^{-s} \text { as } t \downarrow 0 .
$$

Proof. We denote by $E_{p}^{(q, \alpha)}$ the expectation with respect to $X_{t}$ on $[q, 1)$ starting from $p(q \leq p<1)$ with boundary conditions at $q$

$$
f(q) \cos \alpha-f^{\prime}(q) \sin \alpha=0 \text { for } 0<\alpha<\frac{\pi}{2} \quad \text { and } f(1)=0 \text { at } 1 \text {. }
$$

By the strong Markov property,

$$
\begin{aligned}
E_{0}^{(0, \alpha)} e^{-\lambda \tau_{c}}= & E_{0}^{(0, \alpha)} e^{-\lambda \tau_{a_{1}}} E_{a_{1}}^{(0, \alpha)} e^{-\lambda \tau_{b_{1}}} E_{b_{1}}^{(0, \alpha)} e^{-\lambda \tau_{a_{2}} \ldots} \\
& \cdots E_{a_{N}}^{(0, \alpha)} e^{-\lambda \tau_{N}} E_{b_{N}}^{(0, \alpha)} e^{-\lambda \tau_{1}} \\
= & \prod_{i=1}^{N} E_{a_{i}}^{\left(a_{i}, \alpha\right)} e^{-\lambda \tau_{b_{i}} E_{0}^{(0, \alpha)}} e^{-\lambda \tau_{a_{1}}} E_{b_{N}}^{(0, \alpha)} e^{-\lambda \tau_{1}} \prod_{i=1}^{N-1} E_{b_{i}}^{(0, \alpha)} e^{-\lambda \tau_{a_{i+1}}} \\
& \times \prod_{i=1}^{N} \frac{E_{a_{i}}^{(0, \alpha)} e^{-\lambda \tau_{b_{i}}}}{E_{a_{i}}^{\left(a_{i}, \alpha\right)} e^{-\lambda \tau_{b_{i}}}}
\end{aligned}
$$

Here we note that

$$
\text { the topological support of } d m \subset \bigcup_{i=1}^{N} S_{i}([0,1])=\bigcup_{i=1}^{N}\left[a_{i}, b_{i}\right]
$$

i.e.

$$
\begin{aligned}
& m\left(\left(b_{i}, a_{i+1}\right)\right)=0 \quad \text { for } \quad 1 \leq i \leq N-1 \text { and } \\
& m\left(\left[0, a_{i}\right)\right)=m\left(\left(b_{N}, 1\right]\right)=0 .
\end{aligned}
$$

Combining (4.6) and (4.7) with Lemma 4.1 and Lemma 4.2, we deduce that there exists nonnegative constants $D_{i}, E_{i}, F_{i}, G_{i}, H_{i}, I_{i}, J_{i}(1 \leq i \leq N)$ such that, for all $\lambda>0$,

$$
\begin{aligned}
& \prod_{i=1}^{N}\left\{I_{i}+\frac{J_{i}}{\lambda}\right\} \prod_{i=1}^{N} E_{a_{i}}^{\left(a_{i}, \alpha\right)} e^{-\lambda \tau_{b_{i}} \geq E_{0}^{(0, \alpha)} e^{-\lambda \tau_{1}}} \\
& \quad \geq \prod_{i=1}^{N} E_{a_{i}}^{\left(a_{i}, \alpha\right)} e^{-\lambda \tau_{b_{i}}} \prod_{i=1}^{N} \frac{1}{D_{i}+\lambda E_{i}+\frac{F_{i}}{\lambda} \prod_{i=1}^{N+1} \frac{1}{G_{i}+\lambda H_{i}}} .
\end{aligned}
$$

Put

$$
f(\lambda)=E_{0}^{(0, \alpha)} e^{-\lambda \tau_{1}}, \quad f_{1}(\lambda)=\prod_{i=1}^{N}\left\{I_{i}+\frac{J_{i}}{\lambda}\right\} \quad \text { and }
$$




$$
f_{2}(\lambda)=\prod_{i=1}^{N} \frac{1}{D_{i}+\lambda E_{i}+\frac{F_{i}}{\lambda}} \prod_{i=1}^{N+1} \frac{1}{G_{i}+\lambda H_{i}}
$$

Noting the self-similarity of $d m$ and that $\left[a_{i}, b_{i}\right]=S_{i}[0,1]$, we can conclude from (4.8) that

$$
f_{2}(\lambda) \prod_{i=1}^{N} f\left(\rho_{i}\left|r_{i}\right| \lambda\right) \leq f(\lambda) \leq f_{1}(\lambda) \prod_{i=1}^{N} f\left(\rho_{i}\left|r_{i}\right| \lambda\right)
$$

Setting $g(\lambda)=-\log f(\lambda), g_{1}(\lambda)=-\log f_{1}(\lambda), g_{2}(\lambda)=-\log f_{2}(\lambda)$, we obtain that

$$
g_{2}(\lambda)+\sum_{i=1}^{N} g\left(\rho_{i}\left|r_{i}\right| \lambda\right) \geq g(\lambda) \geq g_{1}(\lambda)+\sum_{j=1}^{N} g\left(\rho_{i}\left|r_{i}\right| \lambda\right) .
$$

Take the unique number $u\left(0<u<\frac{1}{2}\right)$ such that

$$
\sum_{i=1}^{N}\left(\rho_{i}\left|r_{i}\right|\right)^{u}=1
$$

Then clearly, $\sum_{i=1}^{N}\left(\rho_{i}\left|r_{i}\right|\right)^{u / 2}>1$ and noting the form of $f_{1}(\lambda)$ and $f_{2}(\lambda)$, we can easily deduce that

$$
\begin{aligned}
& \left(\sum_{i=1}^{N}\left(\rho_{i}\left|r_{i}\right|\right)^{u / 2}-1\right) \lambda^{u / 2}+\sum_{i=1}^{N} g\left(\rho_{i}\left|r_{i}\right| \lambda\right) \geq g(\lambda) \\
& \quad \geq-K_{1}+\sum_{i=1}^{N} g\left(\rho_{i}\left|r_{i}\right| \lambda\right)
\end{aligned}
$$

for all $\lambda \geq \lambda_{0}$ where $K_{1}$ and $\lambda_{0}(>1)$ are suitably chosen positive constants. Putting $k(\lambda)=\frac{g(\lambda)}{\lambda^{u}}$, we set

$$
\begin{aligned}
C_{4.3} & =\min _{\substack{\lambda \in\left[\min \rho_{i}\left|r_{i}\right|, 1\right] \\
1 \leq i \leq N}} k(\lambda), \\
C_{4.4} & =\max _{\substack{\lambda \in\left[\min \rho_{i}\left|r_{i}\right|, 1\right] \\
1 \leq i \leq N}}\left(k(\lambda)+\lambda^{-u / 2}\right) .
\end{aligned}
$$

From the first inequality of (4.10),

$$
k(\lambda)=\frac{g(\lambda)}{\lambda^{u}} \leq \sum_{i=1}^{N}\left(\rho_{i}\left|r_{i}\right|\right)^{u} k\left(\rho_{i}\left|r_{i}\right| \lambda\right)+\left(\sum_{i=1}^{N}\left(\rho_{i}\left|r_{i}\right|\right)^{u / 2}-1\right) \lambda^{-u / 2} .
$$

Let $k_{1}(\lambda)=k(\lambda)+\lambda^{-u / 2}$. Then, for all $\lambda \geq \lambda_{0}(>1)$,

$$
k_{1}(\lambda) \leq \sum_{i=1}^{N}\left(\rho_{i}\left|r_{i}\right|\right)^{u} k_{1}\left(\rho_{i}\left|r_{i}\right| \lambda\right) \leq \max _{1 \leq i \leq N} k_{1}\left(\rho_{i}\left|r_{i}\right| \lambda\right) .
$$

Applying (4.11) successively, 


$$
k(\lambda) \leq k\left(\rho_{i_{1}}\left|r_{i_{1}}\right| \cdots \rho_{i_{M K}}\left|r_{i_{M}}\right|\right)
$$

for some $i_{1}, \cdots, i_{M} \in\{1, \cdots, N\}$ such that

$$
\min _{1 \leq i \leq N} \rho_{i}\left|r_{i}\right| \leq \rho_{i_{1}}\left|r_{i_{1}}\right| \cdots \rho_{i_{\text {MU }}}\left|r_{i_{\text {MU }}}\right| \lambda \leq 1
$$

This proves $\quad k_{1}(\lambda) \leq C_{4.4}$ for all $\lambda \geq \lambda_{0}$.

Similarly, we can prove that

$$
k(\lambda) \geq C_{4.3} \quad \text { for all } \lambda \geq \lambda_{0} .
$$

Therefore, we obtain the following estimate

$$
C_{4.3} \lambda^{u} \leq-\log E_{0}^{(0, \alpha)} e^{-\lambda r_{1}} \leq C_{4.4} \lambda^{u} \quad \text { for all } \lambda \geq \lambda_{0}
$$

where $C_{4.5}$ is another positive constant. Seeing the condition $m([x, y])>0$, there exists $i(1 \leq i \leq N)$ such that $x<a_{i}<y<b_{i}$ or $a_{i}<x<b_{i}<y$ holds. In the case that $x<a_{i}<y<b_{i}$, there exists $n_{1}$ such that

$$
\underbrace{S_{1} \circ \cdots \cdots \cdots \circ S_{1} \circ S_{i}[0,1] \subset[x, y] .}_{n_{1} \text { times }}
$$

In the case that $a_{i}<x<b_{i}<y$, there exists $n_{2}$ such that

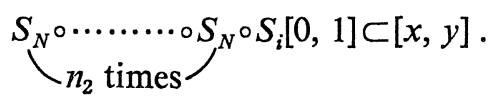

In both cases, there exist some $S_{i_{1}}, \cdots, S_{i_{m}}$ such that

$$
S_{i_{1}} \circ \cdots \circ S_{i_{m}}([0,1]) \subset[x, y] \subset[0,1] .
$$

Combining (4.12) and (4.13) with Lemma 4.1, we deduce that

$$
C_{4.6} \lambda^{u} \leq-\log E_{x}^{(0, \alpha)} e^{-\lambda \tau_{y}} \leq C_{4.4} \lambda^{u} \quad \text { for all } \lambda \geq \lambda_{0}
$$

where $C_{4.6}$ is another positive constant.

Using de Brujin's exponential Tauberian theorem (see [2]), there exist some positive constants $C_{4.1}$ and $C_{4.2}$ such that

$$
C_{4.1} t^{-u /(1-u)} \leq-\log P_{x}\left[\tau_{y} \leq t\right] \leq C_{4.2} t^{-u /(1-u)}
$$

for all small $t>0$ i.e. because of the definition of $s$,

$$
C_{4.1} t^{-s} \leq-\log P_{x}\left[\tau_{y} \leq t\right] \leq C_{4.2} t^{-s} \quad \text { for all small } t>0 .
$$

Noting that $p(t, x, y)=\int_{0}^{t} p(t-s, y, y) P_{x}\left(\tau_{y} \in d s\right)$ and $A:=\min _{0 \leq s \leq t} p(t-s, y, y)>$ 0 , we see that $p(t, x, y) \geq A P_{x}\left[\tau_{y} \leq t\right]$, and hence 


$$
\varlimsup_{t \downarrow 0}-t^{s} \log p(t, x, y) \leq \varlimsup_{t \downarrow 0}-t^{s} \log P_{x}\left[\tau_{y} \leq t\right] \leq C_{4.2} .
$$

On the other hand, taking $c \in K(S)(x<c<y)$, then

$$
M:=\max _{0 \leq s \leq t} p(t-s, c, y)<+\infty .
$$

Hence, $\quad p(t, x, y)=\int_{0}^{t} p(t-s, c, y) P_{x}\left(\tau_{c} \in d s\right) \leq M P_{x}\left[\tau_{c} \leq t\right]$,

i.e. $\quad \frac{\lim }{t \downarrow 0}-t^{s} \log p(t, x, y) \geq \frac{\lim }{t \downarrow 0}-t^{s} \log P_{x}\left[\tau_{c} \leq t\right] \geq C_{4.1}$.

This completes the proof.

If we assume some additional conditions on $S$ and $\rho$, we can obtain a better estimate about $p(t, x, y)$ : It seems an interesting generalization of $\mathrm{S}$. Watanabe's estimate stated in $\S 1$ because of the appearance of the term like a singular Riemannian metric $\hat{F}(x, y)$.

We start with some analysis lemma.

Lemma 4.4. Let $T$ be $a$ bounded continuous function from $(0,+\infty)$ to $(0,+\infty)$ satisfying the following functional equation:

$$
T(\lambda)=p_{1} T\left(q_{1} \lambda\right)+\cdots+p_{n} T\left(q_{n} \lambda\right)
$$

where $p_{i}>0, q_{i}>0$ such that $p_{1}+\cdots+p_{n}=1$ and there exists $i$ and $j$ such that

$$
\frac{\log q_{j}}{\log q_{i}} \notin \boldsymbol{Q}
$$

Then $T$ is a constant function.

Proof. Putting $U(\lambda)=T\left(e^{\lambda}\right)(\lambda \in \boldsymbol{R})$, we have

$$
U(\lambda)=p_{1} U\left(\log q_{1}+\lambda\right)+\cdots+p_{n} U\left(\log q_{n}+\lambda\right) .
$$

Applying the Fourier transform to (4.14) for a slowly increasing distribution $U(\lambda)$, we obtain that

$$
\hat{U}(t)=p_{1} e^{i t \log q_{1}} \hat{U}(t)+\cdots+p_{n} e^{i t \log q_{n}} \hat{U}(t)
$$

where

$$
\hat{U}(t)=\int_{R} e^{i t \lambda} U(\lambda) d \lambda .
$$

Then $1=p_{1} \cos \left(t \log q_{1}\right)+\cdots+p_{n} \cos \left(t \log q_{n}\right)$ on the support of $\hat{U}(t)$. Combining this with the condition on $p_{i}$, we have that $\cos \left(t \log q_{1}\right)=\cdots=\cos \left(t \log q_{n}\right)$ $=1$ on the support of $\hat{U}(t)$. By the assumption on $q_{i}$, we can deduce that the support of $\hat{U}(t)=\{0\}$. Since $U$ is bounded, $U(\lambda)$ must be a constant function. 
Theorem 4.5. Assume that there exist $\rho_{i}\left|r_{i}\right|$ and $\rho_{j}\left|r_{j}\right|$ such that

$$
\frac{\log \rho_{i}\left|r_{i}\right|}{\log \rho_{j}\left|r_{j}\right|} \notin \mathbf{Q}
$$

Then, if we take $x$ and $y$ as in Theorem 4.3, we have that

$$
-\lim _{t \downarrow 0} t^{s} L(t) \log p(t, x, y)=\left\{\hat{F}([x, y]\}^{1+s}\right.
$$

where $L(t)$ is a positive bounded slowly varying function and $\hat{F}$ is the $\left\{S, \rho^{\prime}\right\}$-selfsimilar measure with

$$
\rho^{\prime}=\left(\left(\rho_{1}\left|r_{1}\right|\right)^{s /(1+s)}, \cdots,\left(\rho_{N}\left|r_{N}\right|\right)^{s /(1+s)}\right) .
$$

Proof. Let $g(\lambda)$ be defined as in the proof of Theorem 4.3. Putting $G_{c}(\lambda)=\frac{g(c \lambda)}{g(c)}$ for positive number $c\left(c>c_{0}\right.$ where $c_{0}$ is a positive constant $), G_{c}(\lambda)$ is a positive continuous function. Seeing the proof of Theorem 4.3, we can easily conclude there exists a positive constant $C_{4.7}$ such that

$$
\left|G_{c}(\lambda)\right| \leq \frac{C_{4.4} c^{\alpha} \lambda^{\alpha}}{C_{4.3} c^{\alpha}} \leq C_{4.7} \quad \text { for all } \lambda \in I \text { for any }
$$

bounded closed interval $\mathrm{I}$. Then applying Helly's theorem, there exists $c_{n}$ $\left(c_{n} \uparrow+\infty\right)$ such that $G_{c_{n}}(\lambda)$ converges to an increasing function $G(\lambda)$ at every continuity point of $G(\lambda)$. From (4.9) and Theorem 4.3, we can deduce that $G(\lambda)$ satisfies the following functional equation.

$$
G(\lambda)=G\left(\rho_{1}\left|r_{1}\right| \lambda\right)+\cdots+G\left(\rho_{n}\left|r_{n}\right| \lambda\right)
$$

that is,

$$
\begin{aligned}
\frac{G(\lambda)}{\lambda^{s /(1+s)}} & =\left(\rho_{1}\left|r_{1}\right|\right)^{s /(1+s)} \frac{G\left(\rho_{1}\left|r_{1}\right| \lambda\right)}{\left(\rho_{1}\left|r_{1}\right|\right)^{s /(1+s)} \lambda^{s /(1+s)}}+\cdots+\left(\rho_{N}\left|r_{N}\right|\right)^{s /(1+s)} \\
& \cdot \frac{G\left(\rho_{N}\left|r_{N}\right| \lambda\right)}{\left(\rho_{N}\left|r_{N}\right|\right)^{s /(1+s)} \lambda^{s(1+s)}} .
\end{aligned}
$$

From Lemma 4.4 and $G(1)=1$, we can conclude

$$
G(\lambda)=\lambda^{s /(1+s)} \text { 。 }
$$

This shows that every limit point of $G_{c}(\lambda)(c \rightarrow+\infty)$ is the unique function $\lambda^{s /(1+s)}$, that is,

$$
\lim _{c \rightarrow+\infty} \frac{g(c \lambda)}{g(c)}=\lambda^{s /(1+s)}
$$

Then we see that $g(\lambda)=\lambda^{s /(1+s)} L(\lambda)$ where $L(\lambda)$ is a positive bounded slowly 
varying function.

Consider

$$
\begin{aligned}
\bar{E}(x): & =\varlimsup_{\lambda \rightarrow+\infty} \frac{-\log E_{0}^{(0, \alpha)} e^{-\lambda \tau_{x}}}{-\log E_{0}^{(0, \alpha)} e^{-\lambda \tau_{1}}} \\
& =\varlimsup_{\lambda \rightarrow+\infty} \frac{-\log E_{0}^{(0, \alpha)} e^{-\lambda \tau_{x}}}{\lambda^{s /(1+s)} L(\lambda)}
\end{aligned}
$$

If we take $x\left(a_{1} \leq x \leq b_{1}\right)$, we can deduce that by Lemma 4.1 , Lemma 4.2 and the self-similarity of $d m$,

$$
\begin{aligned}
\bar{E}(x) & =\varlimsup_{\lambda \rightarrow+\infty} \frac{-\log E_{0}^{(0, \alpha)} e^{-\rho_{1}\left|r_{1}\right| \lambda \tau \frac{x-a_{1}}{\left|r_{1}\right|}}}{\lambda^{s /(1+s)} L(\lambda)} \\
& =\varlimsup_{\lambda \rightarrow+\infty} \frac{-\log E_{0}^{(0, \alpha)} e^{-\rho_{1}\left|r_{1}\right| \lambda \tau \frac{x-a_{1}}{\left|r_{1}\right|}} \frac{\left(\rho_{1}\left|r_{1}\right| \lambda\right)^{s /(1+s)} L\left(\rho_{1}\left|r_{1}\right| \lambda\right)}{\lambda^{s /(1+s)} L(\lambda)}}{\left(\rho_{1}\left|r_{1}\right| \lambda\right)^{s /(1+s)} L\left(\rho_{1}\left|r_{1}\right| \lambda\right)} \\
& =\left(\rho_{1}\left|r_{1}\right|\right)^{s / 1+s} \bar{E}\left(\frac{x-a_{1}}{\left|r_{1}\right|}\right) .
\end{aligned}
$$

In the case of $a_{i} \leq x \leq b_{i}$, we have similarly a functional equation for $\bar{E}(x)$ and hence deduce that $\bar{E}(x)$ satisfies the functional equation corresponding to $\left\{S, \rho^{\prime}\right\}$-self-similar measure. Because of the uniqueness of the solution of such functional equation, $\bar{E}(x)$ coincides with $\hat{F}([0, x])$ where $\hat{F}$ is the $\left\{S, \rho^{\prime}\right\}$-selfsimilar measure. In the same manner,

$$
E(x):=\lim _{\lambda \rightarrow+\infty} \frac{-\log E_{0}^{(0, \alpha)} e^{-\lambda \tau_{x}}}{-\log E_{0}^{(0, \alpha)} e^{-\lambda \tau_{1}}}
$$

satisfies the same functional equation. Therefore, we obtain that

$$
\lim _{\lambda \rightarrow+\infty} \frac{-1}{L(\lambda)} \lambda^{-s /(1+s)} \log E_{x}^{(0, \alpha)} e^{-\lambda \tau_{y}}=\hat{F}([x, y]) .
$$

By de Brujin's exponential Tauberian theorem (see [2]), we have that

$$
\lim _{t \downarrow 0}-t^{s} L^{1}(t) \log P_{x}\left[\tau_{y} \leq t\right]=\hat{F}([x, y])^{1+s}
$$

where $L^{1}(t)$ is an another positive bounded slowly varying function. Then by the same argument as in the proof of Theorem 4.3 we complete the proof.

Q.E.D.

Remark. In the case of de Rham measure, the condition of Theorem 4.5 is satisfied if 


$$
\frac{\log \frac{p}{2}}{\log \frac{q}{2}} \notin \boldsymbol{Q}
$$

Using the same method, we can also have a better estimate about an asymptotic order of eigenvalues of $L$.

For $0 \leq \alpha, \beta \leq \frac{\pi}{2}, 0 \leq a<b \leq 1$, consider the following eigenvalue problems of $L=\frac{d}{d m} \frac{d}{d x}$ on $[a, b]$ :

$$
\begin{aligned}
& L f=\lambda f \text { in }(a, b) \\
& f(a) \cos \alpha+\frac{d}{d x} f(a) \sin \alpha=0 \\
& f(b) \cos \beta-\frac{d}{d x} f(b) \sin \beta=0 .
\end{aligned}
$$

We denote the number of eigenvalues not exceeding $\lambda$ by

$$
N_{\alpha, \beta}(\lambda,[a, b]) \text {. }
$$

We put

$$
N_{0,0}=\underline{N}, \quad N_{\pi / 2, \pi / 2}=\bar{N} \text {. }
$$

Then the following are well known:

(1) $0 \leq \bar{N}(\lambda,[a, b])-\underline{N}(\lambda,[a, b]) \leq 2$.

(2) $\underline{N}(\lambda,[a, b]) \leq N_{\alpha, \beta}(\lambda,[a, b]) \leq \bar{N}(\lambda,[a, b])$

(3) For $a<c<b$,

$$
\begin{aligned}
& \bar{N}(\lambda,[a, b]) \leq \bar{N}(\lambda,[a, c])+\bar{N}(\lambda,[c, b]) \\
& \underline{N}(\lambda,[a, b]) \geq \underline{N}(\lambda,[a, c])+\underline{N}(\lambda,[c, b]) .
\end{aligned}
$$

Corollary 4.6. Let $S=\left\{S_{1}, \cdots, S_{N}\right\}$ and $\rho=\left(\rho_{1}, \cdots, \rho_{N}\right)$ satisfying that $S_{i}[0,1] \cap S_{j}[0,1]=$ \{one point $\}$ or $\phi$ for $i \neq j$ and we assume that there exist $\rho_{i}\left|r_{i}\right|$ and $\rho_{j}\left|r_{j}\right|$ such that $\frac{\log \rho_{i}\left|r_{i}\right|}{\log \rho_{j}\left|r_{j}\right|} \notin Q$.

Consider the eigenvalue problem (3.1) and let $\left\{\lambda_{n}\right\}$ be eigenvalues such that $0 \geq \lambda_{1}>\lambda_{2} \geq \lambda_{3}, \cdots$. Then $-\lambda_{n}=n^{(1+s) / s} a_{n}$ where $s$ is the similarity dimension of $m=\mu(S, \rho)$ and $a_{n}$ is a positive bounded slowly varying sequence.

Proof. Noting the self-similarity of $d m$ and that the topological support of $d m \subset \bigcup_{i=1}^{N} S_{i}([0,1])=\bigcup_{i=1}^{N}\left[a_{i}, b_{i}\right]$, 


$$
\begin{aligned}
\bar{N}(\lambda,[0,1]) & \leq \bar{N}\left(\lambda,\left[a_{1}, b_{1}\right]\right)+\cdots+\bar{N}\left(\lambda,\left[a_{N}, b_{N}\right]\right) \\
& =\bar{N}\left(\rho_{1}\left|r_{1}\right| \lambda,[0,1]\right)+\cdots+\bar{N}\left(\rho_{N}\left|r_{N}\right| \lambda,[0,1]\right) \\
\underline{N}(\lambda,[a, b]) & \geq \underline{N}\left(\rho_{1}\left|r_{1}\right| \lambda,[0,1]\right)+\cdots+\underline{N}\left(\rho_{N}\left|r_{N}\right| \lambda,[0,1]\right) .
\end{aligned}
$$

Putting $N(\lambda)=N_{\alpha, \beta}(\lambda,[0,1])$, these show that

$$
C_{1}+\sum_{i=1}^{N} N\left(\rho_{i}\left|r_{i}\right| \lambda\right) \leq N(\lambda) \leq C_{2}+\sum_{i=1}^{N} N\left(\rho_{i}\left|r_{i}\right| \lambda\right)
$$

where $C_{1}$ and $C_{2}$ are some constants. Replacing $\frac{g(c \lambda)}{g(c)}$ by $\frac{N(c \lambda)}{N(c)}$ in the proof of Theorem 4.5, we can deduce that $N(\lambda)=\lambda^{s /(1+s)} L(\lambda)$ where $L(\lambda)$ is a positive bounded slowly varying function. Hence we can conclude that $-\lambda_{n}=n^{(1+s) / s} a_{n}$ where $a_{n}$ is a positive bounded slowly varying sequence.

Q.E.D.

As an application of our theorem, we can make some following remarks. Let $d m(x)$ be the de Rham measure. Let us consider $\frac{d}{d m(x)} \frac{d}{d x}$-diffusion processes $X_{t}$ with suitable boundary conditions at 0 and 1 . Let $g_{\lambda}(x, y)$ be the Green kernel:

$$
g_{\lambda}(x, y)=\int_{0}^{\infty} e^{-\lambda t} p(t, x, y) d t .
$$

Theorem 3.1 tells us that there exist some positive constants $C_{4.8}$ and $C_{4.9}$ such that

$$
C_{4.8} \lambda^{-1 /(1+s)} \leq \int_{0}^{1} g_{\lambda}(x, x) d m(x) \leq C_{4.9} \lambda^{-1 /(1+s)}
$$

where $s$ is the similarity dimension of $d m(x)$ i.e. the number $s$ satisfies that

$$
\left(\frac{p}{2}\right)^{s /(1+s)}+\left(\frac{q}{2}\right)^{s /(1+s)}=1
$$

Although there exists an estimate like (4.15), we can prove that at every binary rational point $x$ (i.e. there exists a natural number $N$ such that $x=\sum_{i=1}^{N} \frac{x_{i}}{2^{i}} x_{i}=0$ or 1), the asymptotic order of $g_{\lambda}(x, x)$ is different from $-\frac{1}{1+s}$.

Proposition 4.7. For every binary rational $x$, there exist some positive constants $C_{4.10}, C_{4.11}$ such that

$$
C_{4.10} \lambda^{-1 /(1+\alpha)} \leq g_{\lambda}(x, x) \leq C_{4.11} \lambda^{-1 /(1+\infty)}
$$

where $\alpha=\min \left(\log _{2} \frac{1}{p}, \log _{2} \frac{1}{q}\right)$. 
Proof. Let us assume $p>q$ and we put $\alpha_{+}=\log _{2} \frac{1}{p}, \alpha_{-}=\log _{2} \frac{1}{q}$. Without loss of generality, we may take $x=\frac{1}{2}$. Considering $m_{+}(\varepsilon)=m\left(\left[\frac{1}{2}, \frac{1}{2}+\varepsilon\right)\right)$ and $m_{-}(\varepsilon)=m\left(\left[\frac{1}{2}-\varepsilon, \frac{1}{2}\right]\right)$, we take $h_{+}(\lambda)$ and $h_{-}(\lambda)$ which are characteristic functions to $m_{+}, m_{-}$respectively in Krein's correspondence. By (3.8),

$$
g_{\lambda}\left(\frac{1}{2}, \frac{1}{2}\right)=\frac{1}{\frac{1}{h_{+}(\lambda)}+\frac{1}{h_{-}(\lambda)}} .
$$

On the other hand, by the definition of de Rham measure, we see that

$$
\begin{aligned}
& q \varepsilon^{-\log q / \log 2} \leq m_{+}(\varepsilon) \leq \varepsilon^{-\log q / \log 2} \\
& p \varepsilon^{-\log p / \log 2} \leq m_{-}(\varepsilon) \leq \varepsilon^{-\log p / \log 2} .
\end{aligned}
$$

Since $c \lambda^{-1 /(1+\infty)}$ is the characteristic function corresponding to $d m(x)=d\left(x^{\infty}\right)$ $(0<\alpha<+\infty)$, we see that from the comparison theorem in $\S 2$, there exist some positive constants $C_{4.12}, C_{4.13}, C_{4.14}, C_{4.15}$ such that

$$
\begin{aligned}
& C_{4.12} \lambda^{-1 /\left(1+\alpha_{+}\right)} \leq h_{+}(\lambda) \leq C_{4.13} \lambda^{-1 /\left(1+\infty_{+}\right)} \\
& C_{4.14} \lambda^{-1 /\left(1+\alpha_{-}\right)} \leq h_{-}(\lambda) \leq C_{4.15} \lambda^{-1 /\left(1+\infty_{-}\right)} .
\end{aligned}
$$

So, (4.16), (4.17) and (4.18) completes the proof.

Q.E.D.

Barlow and Perkins [1] proved that there exists some positive constants $C_{4.16}, C_{4.17}, C_{4.18}$ and $C_{4.19}$ such that

$$
\begin{gathered}
C_{4.16} t^{-d_{s} / 2} \exp \left\{-C_{4.17} \frac{|x-y|^{d_{w} /\left(d_{w}-1\right)}}{t^{1 /\left(d_{w 0}-1\right)}}\right\} \leq p(t, x, y) \\
\leq C_{4.18} t^{-d_{s} / 2} \exp \left\{-C_{4.19} \frac{|x-y|^{d_{w} /\left(d_{w}-1\right)}}{t^{1 /\left(d_{w}-1\right)}}\right\}
\end{gathered}
$$

where $p(t, x, y)$ is the transition probability density of the Brownian motion on the Sierpinskii Gasket and $d_{s}=\frac{\log 9}{\log 5}, d_{w}=\frac{\log 5}{\log 2}$. We can show that any diffusion corresponding to a de Rham measure with some boundary conditions does not satisfy an estimate of this type. Namely, we have:

Proposition 4.8. Let $p(t, x, y)$ be the transition probability density of the de Rham diffusion process (i.e. $\frac{d}{d m(x)} \frac{d}{d x}$-diffusion process with $d m=$ the de Rham measure $\left(0<p<1, p \neq \frac{1}{2}\right)$ with some boundary conditions.) Then, $p(t, x, y)$ can 
not have an estimate of the following type: for every $t_{0}>0$, there exist some positive constants $C_{4.20}, C_{4.21}, C_{4.22}$ and $C_{4.23}$ such that for every $(x, y) \in[0,1] \times[0,1]$ and every $t \in\left(0, t_{0}\right)$,

$$
\begin{gathered}
C_{4.20} t^{-\beta} \exp \left\{-C_{4.21} \frac{\rho(x, y)^{\delta}}{t^{\gamma}}\right\} \leq p(t, x, y) \\
\leq C_{4.22} t^{-\beta} \exp \left\{-C_{4.23} \frac{\rho(x, y)^{\delta}}{t^{\gamma}}\right\}
\end{gathered}
$$

where $\beta, \gamma, \delta$ are some positive constants and $\rho(x, y)$ is some metric on $[0,1]$.

Proof. Assume that (4.19) holds.

Then, substituting $x=y$ in (4.19) and integrating by $e^{-\lambda t} d t$

$$
C_{4.20} \lambda^{\beta-1} \leq g_{\lambda}(x, x) \leq C_{4.22} \lambda^{\beta-1} \quad \text { for all } x .
$$

Integrating this by $d m$, we have that

$$
C_{4.20} \lambda^{\beta-1} \leq \int_{0}^{1} g_{\lambda}(x, x) d m(x) \leq C_{4.22} \lambda^{\beta-1} .
$$

Comparing this with Theorem 3.1, we can conclude that $\beta$ must be equal to $\frac{s}{1+s}$.

On the other hand, if $x$ is a binary rational, Proposition 4.7 shows that there exist some positive constants $C_{4.24}$ and $C_{4.25}$

$$
\begin{gathered}
C_{4.24} \lambda^{-1 /(1+\alpha)} \leq g_{\lambda}(x, x) \leq C_{4.25} \lambda^{-1 /(1+\alpha)} \\
\alpha=\max \left(\log _{2} \frac{1}{p}, \log _{2} \frac{1}{q}\right) .
\end{gathered}
$$

where

(4.20) and (4.21) lead a contradiction.

Q.E.D.

\section{References}

[1] Barlow, M.T. and Perkins, E.A., Brownian motion on the Sierpinskii gasket, Probab. Th. Rel. Fields, 79 (1988), 543-623.

[2] Bingham, N.H., Goldie, C.M., Teugels, J.L., Regular Variation, Encyclopedia of mathematics and its applications, Cambridge University Press, 1987.

[3] Fujita, T., A Fractional Dimension, Self-similarity and a Generalized diffusion Operator, Probabilistic Methods in Mathematical Physics (K. Ito and N. Ikeda ed.) Proceedings of Taniguchi International Symposium Katata and Kyoto, 1985, Kinokuniya, (1987), 83-90.

[4] Hong, I. and Uno, T., Some consideration of asymptotic distribution of eigenvalues for the equation $\frac{d^{2} u}{d x^{2}}+\lambda \rho(x) u=0$, Japanese Journal of Math., 29 (1959), 152-164. 
[5] Hutchinson, J.E., Fractals and self-similarity, Indiana Univ. Math. J., 30 (1981), 152-164.

[6] Ito, K. and Mckean Jr., H.P., Diffusion processes and their sample paths, SpringerVerlag, Berlin, 1965.

[7] Kac, I.S., Generalization of an asymptotic formula of V.A. Marchenko for spectral functions of a second order boundary problems with conditions at a regular end, Math. USSR. Izv., 5 (1971), 161-191 (English transl.).

[8] Kinney, J.R., Singular functions associated with Markov chains, Proc. Amer. Math. Soc., 9 (1958), 603-608.

[9] Kotani, S. and Watanabe, S., Krein's spectral theory of strings and generalized diffusion processes, Functional analysis in Markov Processes (ed. by M. Fukushima), The Proceeding of Katata Symposium 1981, Lect. Note in Math., 923 (1982), 235-259.

[10] Krein, M.G., On a generalization of Stieltjes, Dokl. Akad. Nauk. SSSR, 87 (1952), 881-884 (in Russian).

[11] Mandelbrot, B.B., The fractal geometry of nature, Freedman, 1982.

[12] McKean, H.P. and Ray, D.B., Spectral distribution of a differential operator, Duke Math. J., 29 (1962), 281-292.

[13] de Rham, G., Sur quelques courbes definites par des equations fonctionelles, Rend. Sem. Mat. Torino, 16 (1957), 101-113. 